\title{
Enhancing discrete-modulated continuous-variable measurement-device-independent quantum key distribution via quantum catalysis
}

\author{
Wei $\mathrm{Ye}^{1}$, Ying Guo ${ }^{1}$, Yun $\mathrm{Mao}^{2 *}$, Hai Zhong ${ }^{1 \dagger}$ and Liyun $\mathrm{Hu}^{3 \ddagger}$ \\ ${ }^{1}$ School of Computer Science and Engineering, Central South University, Changsha 410083, China \\ ${ }^{2}$ School of Automation, Central South University, Changsha 410083, China \\ ${ }^{3}$ Center for Quantum Science and Technology, Jiangxi Normal University, Nanchang 330022, China \\ *†‡Corresponding authors: maoyun3106@sina.com,zhonghai@csu.edu.cn and hlyun2008@126.com
}

\begin{abstract}
The discrete modulation can make up for the shortage of transmission distance in measurementdevice-independent continuous-variable quantum key distribution (MDI-CVQKD) that has an unique advantage against all side-channel attacks but also challenging for the further performance improvement. Here we suggest a quantum catalysis (QC) approach for enhancing the performance of the discrete-modulated (DM) MDI-CVQKD in terms of the achievable secret key rate and lengthening the maximal transmission distance. The numerical simulation results show that the QC-based MDI-CVQKD with discrete modulation that involves a zero-photon catalysis (ZPC) operation can not only obtain a higher secret key rate than the original DM protocol, but also contributes to the reasonable increase of the corresponding optimal variance. As for the extreme asymmetric and symmetric cases, the secret key rate and maximal transmission distance of the ZPC-involved DM MDI-CVQKD system can be further improved under the same parameters. This approach enables the system to tolerate lower reconciliation efficiency, which will promote the practical implementations with state-of-art technology.
\end{abstract}

\section{INTRODUCTION}

Quantum key distribution (QKD) [1-4], as one of the most mature applications in cryptography, aims to share secret keys between two distant legitimate users (Alice and Bob) even in the presence of an eavesdropper (Eve) [5-7]. Thanks to the usage of efficient detection schemes, including the homodyne and heterodyne detections, the continuous variable (CV) QKD systems [8-12] not only promise high key rates, but also can be easily compatible with the current optical communication technologies. In particular, since the Gaussian-modulated (GM) CVQKD protocol [9] has been theoretically proved to be secure against collective attacks [13] and coherent attacks [14], this protocol paves the way for the development of commercial applications. For example, the field tests of the CVQKD system over $50 \mathrm{~km}$ commercial fiber have been achieved in recent years [15]. Whereas, in practical implementations, the existence of imperfect detectors may lead to the potential security loopholes that could be successfully exploited by Eve to take some attack strategies, e.g., the local oscillator calibration attack [16], the wavelength attack [17], and the detector saturation attack [18], which are still problems for realizing the practical CVQKD system.

In response to these problems, the measurementdevice-independent (MDI) CVQKD protocols were proposed [21-29], where the secret keys between Alice and Bob can be extracted by relying on the measurement results of an untrusted third party (Charlie). This protocol can be immune to all attacks on detectors, and its practical security proof has been analyzed in both the finitesize effect [26, 27] and the composable security [28]. However, in contrast to its discrete-variable (DV) counterpart [19, 20], this MDI-CVQKD has limitations in the transmission distance, which is not sufficient for the re- quirements of the long-distance communication. Therefore, it is an urgent task to lengthen the maximal transmission distance with underlying technology.

Up till now, most of investigations have focused on performance improvement of the MDI-CVQKD system by means of quantum operations [30-32], such as the phase-sensitive optical amplifier and the photon subtraction. To be more specific, using a phase-sensitive optical amplifier to compensate the imperfection of the Bell-state measurement (BSM) implemented by Charlie can improve the performance of the MDI-CVQKD system [30]. In addition, the photon subtraction operations [31, 32] have been used for lengthening the maximal transmission distance of the MDI-CVQKD system, and meanwhile these photon-subtracted operations can be emulated by the non-Gaussian postselection in order to circumvent the complexity of configurations. Despite existing the aforementioned advantages, the success probability of implementing photon-subtracted operations is less than 0.25 , which would lead to the limited performance improvements [33, 34]. To eliminate this drawback, recently, the quantum catalysis [33-38] has been viewed as another useful method to extend the maximal transmission distance of the MDI-CVQKD system [39], compared with the single-photon subtraction case. Unfortunately, a major problem, common to the aforementioned GM CVQKD, is that the reconciliation efficiency $\beta$ is low, especially on the long-distance transmission with a low signal-to-noise ratio.

To solve this problem, there are two approaches. One is to design effective reconciliation code, such as lowdensity parity-check code [40] and multidimensional reconciliation code [41], but this kind of design has a higher cost of hardwares and is hard to be realized in practice, and the alternative is to employ the discretemodulated (DM) schemes [42-49], including the fourstate protocol and the eight-state protocol, which allows 
the distribution of secret keys over long distances in the regime of low signal-to-noise ratio with an efficient reconciliation efficiency. Recently, it has been shown [46] that the DM-based MDI-CVQKD under the extreme asymmetric case rather than the symmetric case has an advantage over the GM-based one with respect to the maximal transmission distance. Motivated by the advantages of the DM schemes with an efficient reconciliation efficiency, in this paper, we put forward a feasible method using a zero-photon catalysis (ZPC) operation in order to further enhance the performance of MDI-CVQKD with discrete modulation. Our results under the same accessible parameters show that, as for the extreme asymmetric and symmetric cases, the usage of the ZPC operation on MDI-CVQKD with discrete modulation can not only give birth to the high secret key rates, but also contributes to the reasonable increase of the corresponding optimal variance. Furthermore, the maximal transmission distance can be lengthened when comparing with the traditional DM scheme. More interestingly, our protocol enables the MDI-CVQKD with discrete modulation to tolerate lower reconciliation efficiency.

The rest of this paper is structured as follows. In Sec. II, we first review the entanglement-based (EB) version of the original DM MDI-CVQKD protocol and then demonstrate the effects of the ZPC operation on MDICVQKD with discrete modulation in detail. In Sec. III, according to the optimality of Gaussian attack, we derive the asymptotic secret key rate of the ZPC-involved MDI-CVQKD with discrete modulation. In Sec. IV, the simulation results and discussions are provided. Finally, our conclusions are drawn in Sec. V.

\section{ZPC-INVOLVED MDI-CVQKD PROTOCOL WITH DISCRETE MODULATION}

The EB version of MDI-CVQKD with discrete modulation is shown in Fig. 1(a), where Alice and Bob respectively generate a two-mode entangled state $\left|\Phi_{4}\right\rangle_{A A_{1}}$ and $\left|\Phi_{4}\right\rangle_{B B_{1}}$, and then both of them respectively hold modes $A$ and $B$ while sending modes $A_{1}$ and $B_{1}$, along with the quantum channels of lengths $L_{A C}$ and $L_{B C}$, to an untrusted third party Charlie who proceeds to interfere with the incoming modes $A_{1}$ and $B_{1}$ at a symmetric beam splitter, and attains two output modes $C_{1}$ and $C_{2}$. After the $x(p)$ quadrature of mode $C_{1}\left(C_{2}\right)$ is measured by the BSM, Charlie proclaims the measured results $\left\{X_{C_{1}}, P_{C_{2}}\right\}$. Subsequently, Bob modifies mode $B$ to $\widetilde{B}$ by using a displacement operation $D(\gamma)$ according to the public results $\left\{X_{C_{1}}, P_{C_{2}}\right\}$, where $\gamma=$ $g\left(X_{C_{1}}+i P_{C_{2}}\right)$ with the gain $g$ of a displacement operation. Through heterodyne detections, Alice and Bob respectively measure mode $A$ and $\widetilde{B}$ to attain the outcome results $\left\{X_{A}, P_{A}\right\}$ and $\left\{X_{B}, P_{B}\right\}$, and finally both of them have to implement the postprocessing to get a string of secret keys.

In what follows, we focus on the ZPC-involved MDI-
CVQKD with discrete modulation. For the sake of discussions, here we take the EB version of the four-state protocol into account. As shown in Fig. 1(b), in the Alice's station, Alice prepares for the two-mode entangled state $\left|\Phi_{4}\right\rangle_{A A_{1}}$ on modes $A$ and $A_{1}$ with a modulated variance $V_{M}=2 \alpha^{2}=V_{A}-1$ given by

$$
\begin{aligned}
\left|\Phi_{4}\right\rangle_{A A_{1}} & =\sum_{l=0}^{3} \sqrt{\lambda_{l}}\left|\phi_{l}\right\rangle_{A}\left|\phi_{l}\right\rangle_{A_{1}} \\
& =\frac{1}{2} \sum_{l=0}^{3} \sqrt{\lambda_{l}}\left|\varphi_{l}\right\rangle_{A}\left|\alpha_{l}\right\rangle_{A_{1}},
\end{aligned}
$$

where the non-Gaussian orthogonal state $\left|\varphi_{l}\right\rangle_{A}$ on mode $A$ is expressed as

$$
\left|\varphi_{l}\right\rangle_{A}=\frac{1}{2} \sum_{j=0}^{3} e^{i(2 l+1) j \pi / 4}\left|\phi_{j}\right\rangle_{A},(l=0,1,2,3),
$$

with

$$
\begin{gathered}
\left|\phi_{l}\right\rangle=\frac{e^{-\alpha^{2} / 2}}{\sqrt{\lambda_{l}}} \sum_{m=0}^{3} \frac{(-1)^{m} \alpha^{4 m+l}}{\sqrt{(4 m+l) !}}|4 m+l\rangle, \\
\lambda_{0,2}=\frac{e^{-\alpha^{2}}}{2}\left[\cosh \alpha^{2} \pm \cos \alpha^{2}\right], \\
\lambda_{1,3}=\frac{e^{-\alpha^{2}}}{2}\left[\sinh \alpha^{2} \pm \sin \alpha^{2}\right],
\end{gathered}
$$

and $\left|\alpha_{l}\right\rangle_{A_{1}}=\left|\alpha e^{i(2 l+1) \pi / 4}\right\rangle(l=0,1,2,3)$ on mode $A_{1}$ is a modulated coherent state with a positive number $\alpha$. Note that, one of the states $\left|\alpha_{l}\right\rangle_{A_{1}}$ with the same probability $1 / 4$ is randomly sent to Charlie through an unsecure quantum channel. Thus, the corresponding covariance matrix $\Gamma_{A A_{1}}$ of the resending state $\left|\Phi_{4}\right\rangle_{A A_{1}}$ is

$$
\Gamma_{A A_{1}}=\left(\begin{array}{cc}
X I_{2} & Z_{4} \sigma_{z} \\
Z_{4} \sigma_{z} & X I_{2}
\end{array}\right),
$$

where $I_{2}=\operatorname{diag}(1,1), \sigma_{z}=\operatorname{diag}(1,-1)$, and

$$
X=1+2 \alpha^{2}, Z_{4}=2 \alpha^{2} \sum_{k=0}^{3} \lambda_{k-1}^{3 / 2} \lambda_{k}^{-1 / 2} .
$$

In order to lower requirements for device perfection of the quantum catalysis, we assume that, for our protocol, an untrusted third party, David, who is near Alice, controls the ZPC operation (magenta box) where a vacuum state $|0\rangle_{D}$ in an auxiliary mode $D$ is injected into the beam splitter (BS) with a transmittance $T$, and simultaneously an ideal on/off detector placed at the output port of mode $D$ is used for registering the same state $|0\rangle_{D}$. As shown in Refs. [33, 34], the vacuum state $|0\rangle_{D}$ between the input and output ports seems to be unchanged, but promotes the transformation of quantum states between 


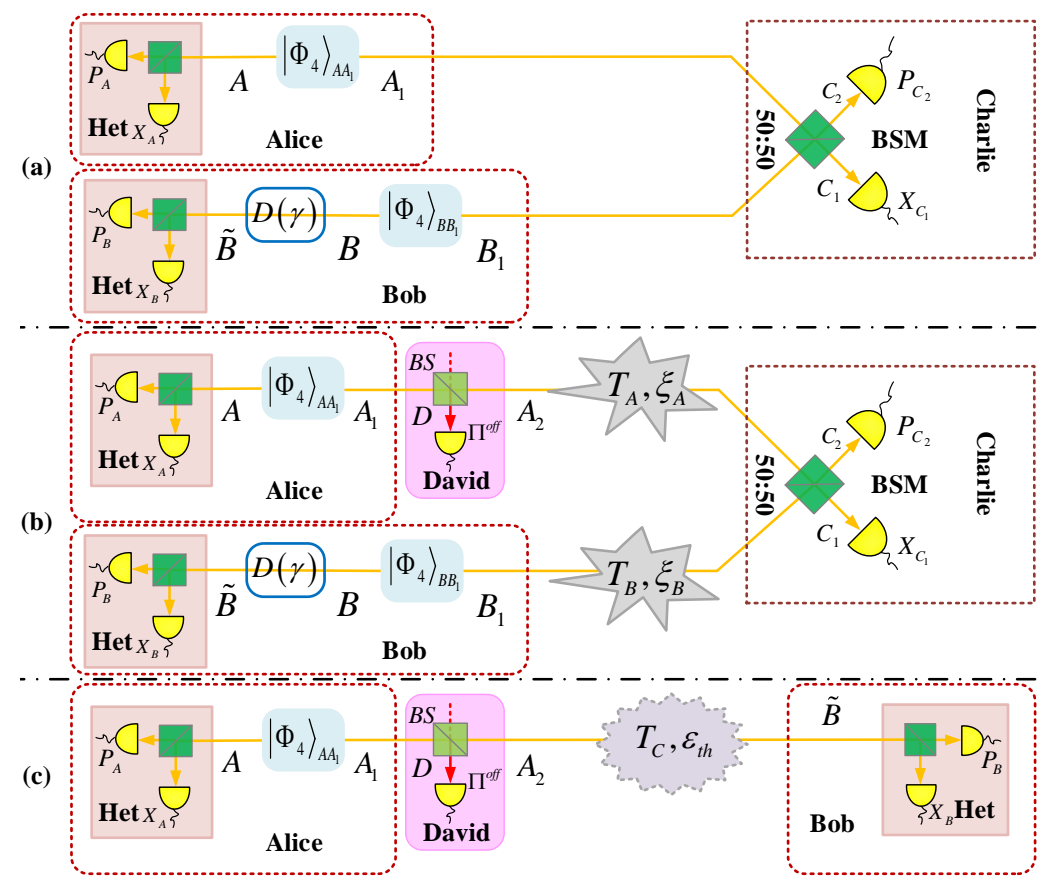

FIG. 1: (Color online) (a) EB version of the original MDI-CVQKD with discrete modulation. (b) EB version of the ZPC-involved MDI-CVQKD with discrete modulation. (c) Equivalent one-way CVQKD protocol with the ZPC operation under the assumption that Bob's prepared state $\left|\Phi_{4}\right\rangle_{B B_{1}}$ and displacement except for heterodyne detection are untrusted. Both $\left|\Phi_{4}\right\rangle_{A A_{1}}$ and $\left|\Phi_{4}\right\rangle_{B B_{1}}$ are the two-mode entangled states. $50: 50$ is a balanced beam splitter. BS is a beam splitter with a transmittance $T$. BSM is a Bell-state measurement. Het and Hom are respectively a heterodyne detection and a homodyne detection. $\left\{X_{j}, P_{j}\right\}(j=A, B)$ is the outcome results of the heterodyne detection. $X_{C_{1}}$ (or $P_{C_{2}}$ ) is the outcome results of the BSM. $D(\gamma)$ is a displacement operator. $\Pi^{o f f}=|0\rangle_{D}\langle 0|$ is a projective operator. $T_{j}$ and $\xi_{j}(j=A, B)$ are respectively the quantum channel transmittance and the excess noise. $T_{C}$ and $\varepsilon_{t h}$ are respectively the equivalent quantum channel transmittance and excess noise of the equivalent one-way protocol.

modes $A_{1}$ and $A_{2}$. Moreover, this ZPC operation can be described as an equivalent operator

$$
\widehat{O}_{0}=\operatorname{Tr}\left[\Pi^{o f f} B(T)\right]=\sqrt{T}^{b^{\dagger} b}
$$

where $B(T)=\exp \left[\left(a d^{\dagger}-a^{\dagger} d\right) \arccos \sqrt{T}\right]$ is a BS operator and $\Pi^{o f f}=|0\rangle_{D}\langle 0|$ is a projective operator on mode $D$. In addition, when such an operation is applied to an arbitrary coherent state $|\alpha\rangle$, one can obtain

$$
\begin{aligned}
|\psi\rangle_{\text {out }} & =\frac{\widehat{O}_{0}}{\sqrt{P_{d}}}|\alpha\rangle \\
& =\frac{\exp \left[-\frac{|\alpha|^{2}}{2}(1-T)\right]}{\sqrt{P_{d}}}|\sqrt{T} \alpha\rangle,
\end{aligned}
$$

where $P_{d}=\exp \left[-|\alpha|^{2}(1-T)\right]$ presents the success probability of implementing such an operation. Evidently, with the assistant of the ZPC, the conversion of quantum states from $|\alpha\rangle$ to $|\sqrt{T} \alpha\rangle$ can be achieved with the probability $P_{d}$. Thus, when one mode $A_{1}$ of the states $\left|\Phi_{4}\right\rangle_{A A_{1}}$ is sent by Alice, the travelling state after succeeding in the ZPC operation can be expressed as $\left|\Phi_{4}\right\rangle_{A A_{2}}=\sum_{l=0}^{3} \sqrt{\lambda_{l}} / 2\left|\varphi_{l}\right\rangle_{A}\left|\widetilde{\alpha}_{l}\right\rangle_{A_{2}}$ with a covariance matrix

$$
\Gamma_{A A_{2}}=\left(\begin{array}{cc}
\widetilde{X} I_{2} & \widetilde{Z}_{4} \sigma_{z} \\
\widetilde{Z}_{4} \sigma_{z} & \widetilde{X} I_{2}
\end{array}\right),
$$

where $\widetilde{X}$ and $\widetilde{Z}_{4}$ can be obtained by replacing $\alpha$ with $\widetilde{\alpha}$ in Eq. $(5)$, and $\left|\widetilde{\alpha}_{l}\right\rangle_{A_{2}}=\left|\widetilde{\alpha} e^{i(2 l+1) \pi / 4}\right\rangle_{A_{2}}$ with $\widetilde{\alpha}=\sqrt{T} \alpha$. It should be noted that after performing the ZPC operation, the actual modulated variance of the travelling state $\left|\Phi_{4}\right\rangle_{A A_{2}}$ turns out to be $\widetilde{V}_{M}=T\left(V_{A}-1\right)$. At the Bob's station, for simplicity, we assume that the variance of the two-mode entangled state $\left|\Phi_{4}\right\rangle_{B B_{1}}$ is the same as that of the state $\left|\Phi_{4}\right\rangle_{A A}$, i.e., $V_{A}=V_{B}=V$ throughout this paper. Besides, due to the fact that Alice and Bob carry out the same discrete modulation, the way of attaining the covariance matrix $\Gamma_{B B_{1}}$ is the same as the Eq. (5).

\section{THE SECRET KEY RATE OF THE ZPC-INVOLVED MDI-CVQKD SYSTEM}

So far, we have established the schematic structure of the EB version of the ZPC-involved MDI-CVQKD system with discrete modulation. In this section, for the reverse reconciliation algorithm, we pay attention to the 


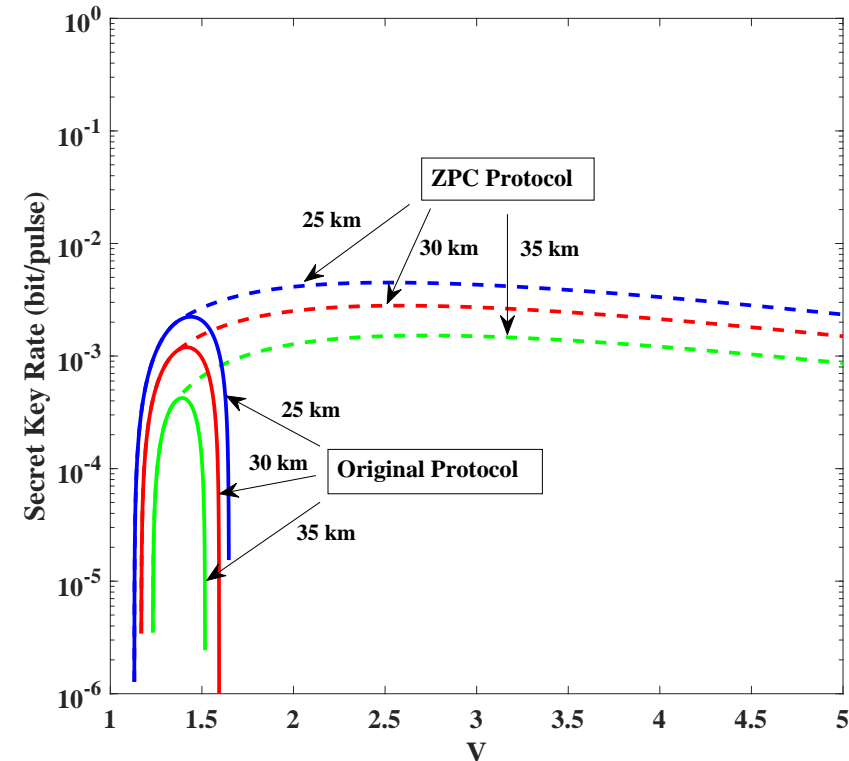

(a)

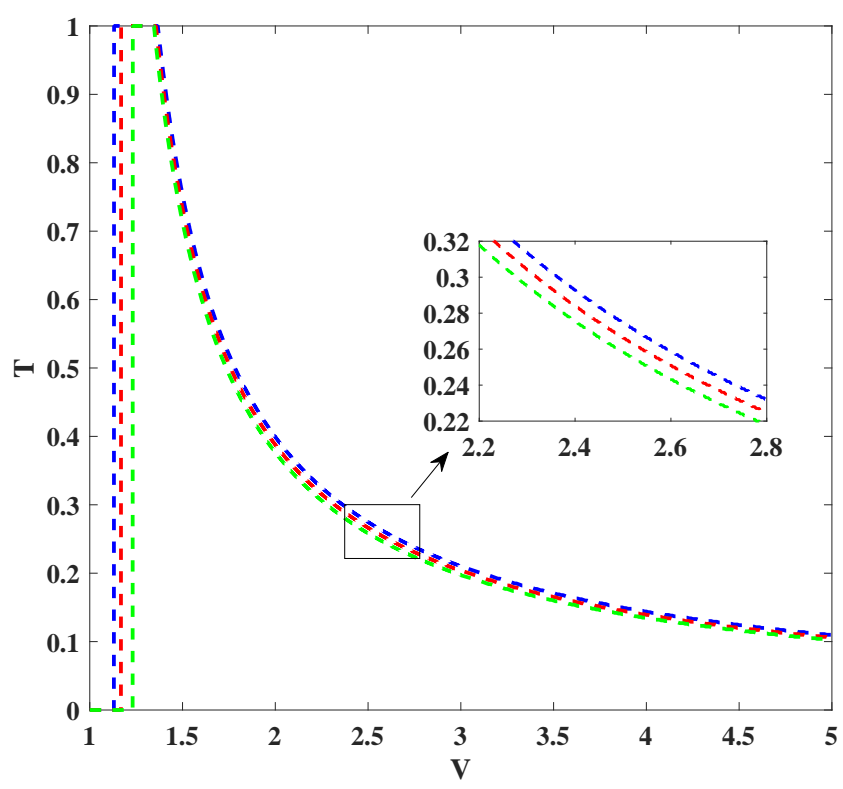

(c)

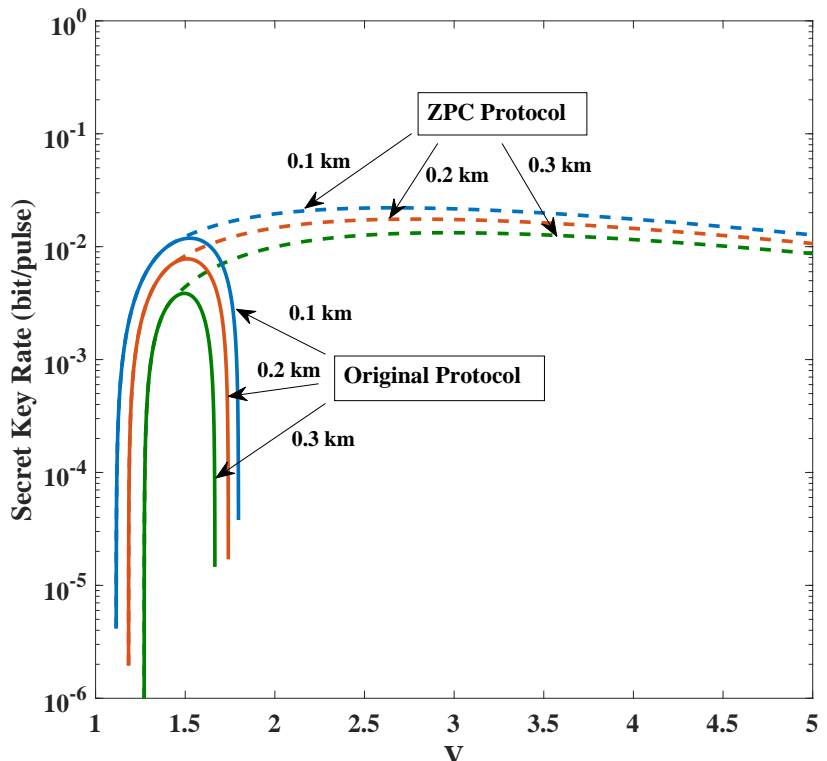

(b)

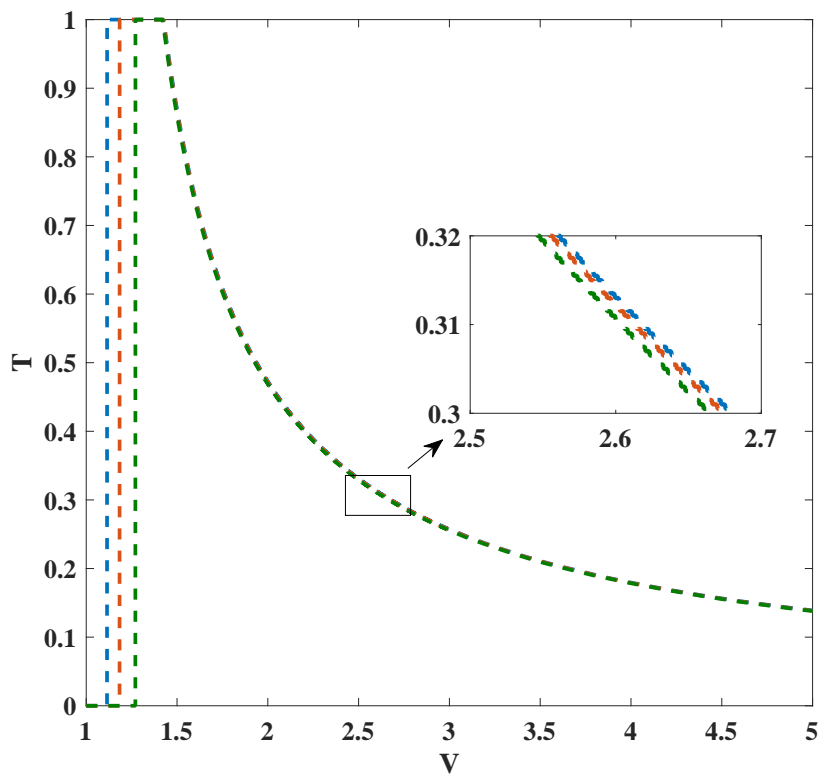

(d)

FIG. 2: (Color online) The secret key rate versus the variance $V$ for (a) the extreme asymmetric case and (b) the symmetric case with transmission distances $\{25 \mathrm{~km}, 30 \mathrm{~km}, 35 \mathrm{~km}\}$ and $\{0.1 \mathrm{~km} 0.2 \mathrm{~km}, 0.3 \mathrm{~km}\}$ when optimizing over the transmittance $T$ shown in (c) and (d), respectively. Note that ZPC Protocol and Original Protocol represent the ZPC-involved MDI-CVQKD and the original MDI-CVQKD with discrete modulation. Other parameters are given by reconciliation efficiency $\beta=0.95$ and excess noise $\xi_{j}=\xi=0.002$ for $j \in\{A, B\}$

derivation of asymptotic secret key rates against onemode collective Gaussian attacks [13, [50] where two Markovian memoryless Gaussian quantum channels are not related [51]. Here we consider the results that the both channels between Alice (or Bob) and Charlie are Gaussian under the condition of the modulated variance $V_{M}=V-1<0.5$ [42 46], which arises from the indistinguishability of $Z_{4}$ and $Z_{G}=\sqrt{V^{2}-1}$. It means that the DM scheme can be viewed as the GM one when satisfying the condition of $V_{M}<1.5$. In Fig. 1(b), we assume that the losses of both channels are $\kappa=0.2 \mathrm{~dB} / \mathrm{km}$, while $T_{A}=10^{-\kappa L_{A C} / 10}\left(T_{B}=10^{-\kappa L_{B C} / 10}\right)$ and $\xi_{A}\left(\xi_{B}\right)$ respectively represent the transmittance and excess noise of the channel between Alice (Bob) and Charlie. As referred to Refs. [21-28, 30-32], the EB version of the MDI-CVQKD system can be equivalent to a conventional 
one-way CVQKD system with the assumption that both the state $\left|\Phi_{4}\right\rangle_{B B_{1}}$ and the displacement operation $D(\gamma)$ in Bob's station are untrusted except for the heterodyne detection, as shown in Fig. 1(c). For the equivalent system, $T_{C}$ and $\varepsilon_{t h}$ are respectively the equivalent quantum channel transmittance and excess noise through the relations

$$
\begin{aligned}
T_{C} & =\frac{g^{2} T_{A}}{2}, \\
\varepsilon_{t h} & =1+\chi_{A}+\frac{T_{B}}{T_{A}}\left(\chi_{B}-1\right) \\
& +\frac{T_{B}}{T_{A}}\left(\sqrt{\frac{2\left(V_{B}-1\right)}{g^{2} T_{B}}}-\sqrt{V_{B}+1}\right)^{2},
\end{aligned}
$$

where $\chi_{j}=\left(1-T_{j}\right) / T_{j}+\xi_{j}(j=A, B)$. Further, by setting $g^{2}=2\left(V_{B}-1\right) /\left[\left(V_{B}+1\right) T_{B}\right]$, one can derive the minimum $\varepsilon_{t h}$, i.e.,

$$
\varepsilon_{t h}=\frac{T_{B}}{T_{A}}\left(\chi_{B}-1\right)+1+\chi_{A} .
$$

Although the imperfection of the BSM in Charlie is an inescapable event, such an imperfection can be compensated by optical preamplifiers [32, 52, 53]. For simplicity, we consider the assumption of the perfection detection for performance analysis. Consequently, the total noise of the channel input in the shot-noise units (SNU) can be expressed as $\chi_{t}=\left(1-T_{C}\right) / T_{C}+\varepsilon_{t h}$.

According to the aforementioned analysis, after performing the BSM and the displacement operation, the covariance matrix $\Gamma_{A \widetilde{B}}$ of the state $\left|\Phi_{4}\right\rangle_{A \widetilde{B}}$ is

$$
\begin{aligned}
\Gamma_{A \widetilde{B}} & =\left(\begin{array}{ll}
a I_{2} & c \sigma_{z} \\
c \sigma_{z} & b I_{2}
\end{array}\right) \\
& =\left(\begin{array}{cc}
\widetilde{X} I_{2} & \sqrt{T_{C}} \widetilde{Z}_{4} \sigma_{z} \\
\sqrt{T_{C}} \widetilde{Z}_{4} \sigma_{z} & T_{C}\left(\widetilde{X}+\chi_{t}\right) I_{2}
\end{array}\right) .
\end{aligned}
$$

We note that the ZPC operation belongs to a kind of Gaussian operation, which makes it possible to derive the secret key rates by using the results of the extremality of Gaussian quantum states [54]. Thus, we have the secret key rate of the ZPC-involved MDI-CVQKD system with discrete modulation under reverse reconciliation against one-mode collective Gaussian attacks, i.e.,

$$
K=P_{d}\{\beta I(A: B)-\chi(B: E)\},
$$

where the success probability $P_{d}$ has been given in Eq. (8), $\beta$ denotes a reverse-reconciliation efficiency, $I(A: B)$ denotes the Shannon mutual information between Alice and Bob, which can be derived as

$$
I(A: B)=\log _{2} \frac{a+1}{a+1-c^{2} /(Y+1)},
$$

and $\chi(B: E)$ denotes the Holevo bound between Bob and Eve. To obtain this Holevo bound, we assume that Eve is aware of the untrusted third party David, and can purify the whole system $\rho_{A \widetilde{B} E D}$, so that

$$
\begin{aligned}
\chi(B: E) & =S(E)-S(E \mid B) \\
& =S(A \widetilde{B})-S\left(A \mid \widetilde{B}^{m_{B}}\right), \\
& =\sum_{i=1}^{2} G\left[\frac{\lambda_{i}-1}{2}\right]-G\left[\frac{\lambda_{3}-1}{2}\right],
\end{aligned}
$$

where $G[x]=(x+1) \log _{2}(x+1)-x \log _{2} x, S(A \widetilde{B})$ is a function of the symplectic eigenvalues $\lambda_{1,2}$ of $\Gamma_{A \widetilde{B}}$ given by

$$
\lambda_{1,2}^{2}=\frac{1}{2}\left[\vartheta \pm \sqrt{\vartheta^{2}-4 \zeta^{2}}\right],
$$

with $\vartheta=a^{2}+b^{2}-2 c^{2}$ and $\zeta=a b-c^{2}$, and Eve's condition entropy $S\left(A \mid \widetilde{B}^{m_{B}}\right)$ based on Bob's measurement result $m_{B}$, is a function of the symplectic eigenvalues $\lambda_{3}$ of $\Gamma_{A}^{m_{B}}=a I_{2}-c \sigma_{z}\left(Y I_{2}+I_{2}\right)^{-1} c \sigma_{z}$, which is given by

$$
\lambda_{3}=\frac{a(b+1)-c^{2}}{b+1} .
$$

\section{NUMERICAL SIMULATIONS AND DISCUSSIONS}

In the traditional MDI-CVQKD system, most of investigations show that the extreme asymmetric case has the best performance when comparing with the symmetric case $\left(L_{A C}=L_{B C}\right)$ [21-28, 30,-32]. That is, if Charlie is close to Bob, then the extreme asymmetric case $\left(L_{B C}=0\right)$ is more suitable for the point-to-point communication in contrast to the symmetric case, enabling us to achieve the maximal transmission distance. Different from the former, the latter has an unique shortdistance network application, e.g., the quantum repeater where the relay (Charlie) has to be placed in the middle of the legitimate communication parties. To extract the maximal secret key rates as many as possible, in the following, we demonstrate the optimal area and value of the variance $V$ that is an important factor of affecting the performance of MDI-CVQKD systems with discrete modulation, and then proceed with the security analysis, including the extreme asymmetric and symmetric cases.

\section{A. Parameter optimization}

Now, let us examine the optimal area and value of $V$ in the ZPC-involved DM MDI-CVQKD system, which determines the transmitting power of the quantum signal, thereby impacting the performance of the CVQKD systems. We note that the actual modulated variance $\widetilde{V}_{M}$ of the proposed system is expressed as $\widetilde{V}_{M}=\widetilde{V}-1=$ $T(V-1)$ according to the relation of $\widetilde{\alpha}=\sqrt{T} \alpha$, which 


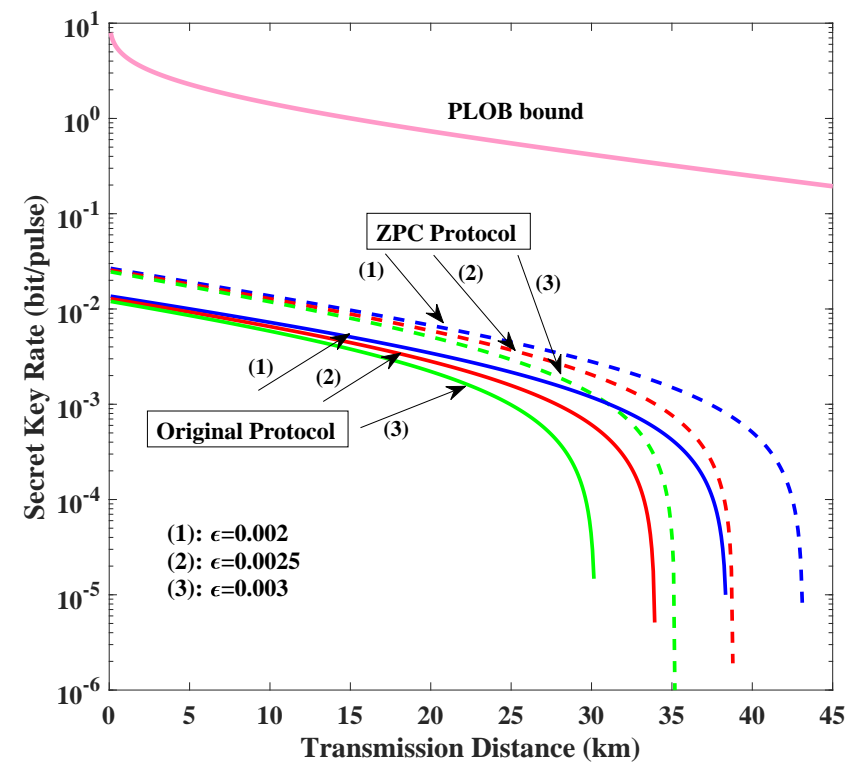

(a)

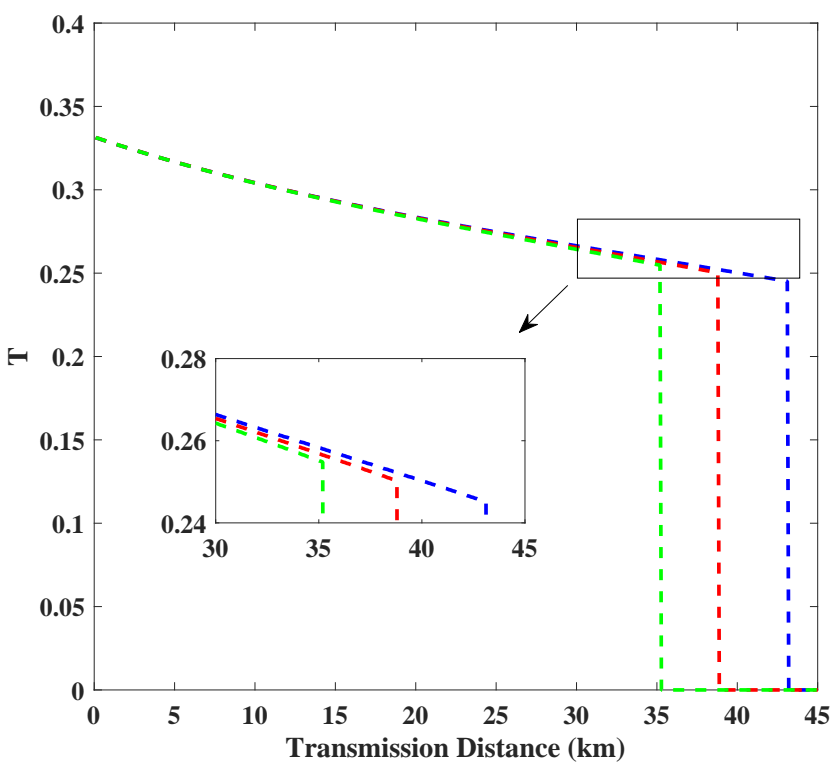

(c)

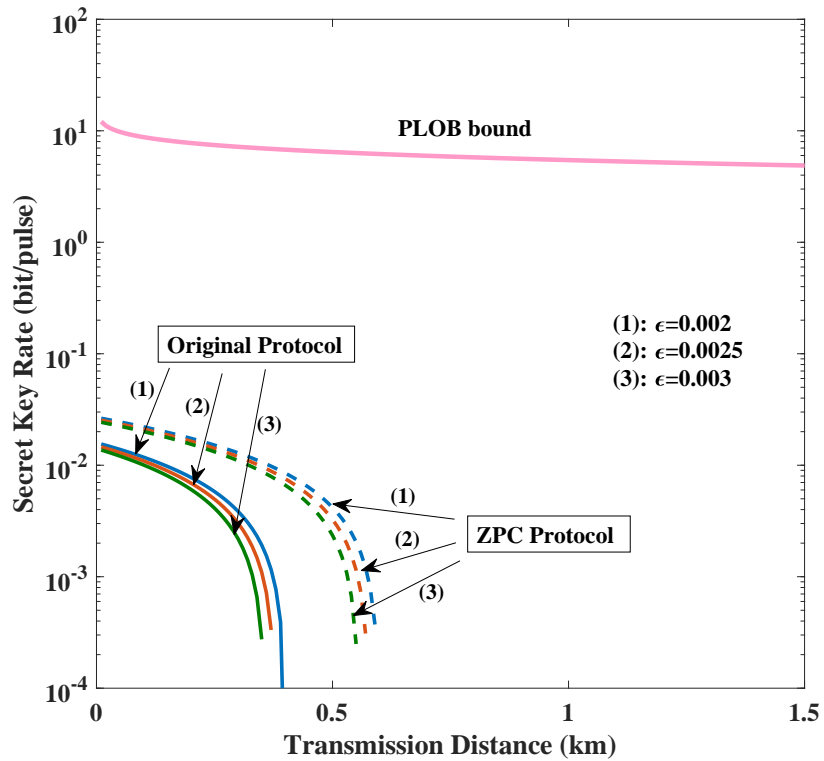

(b)

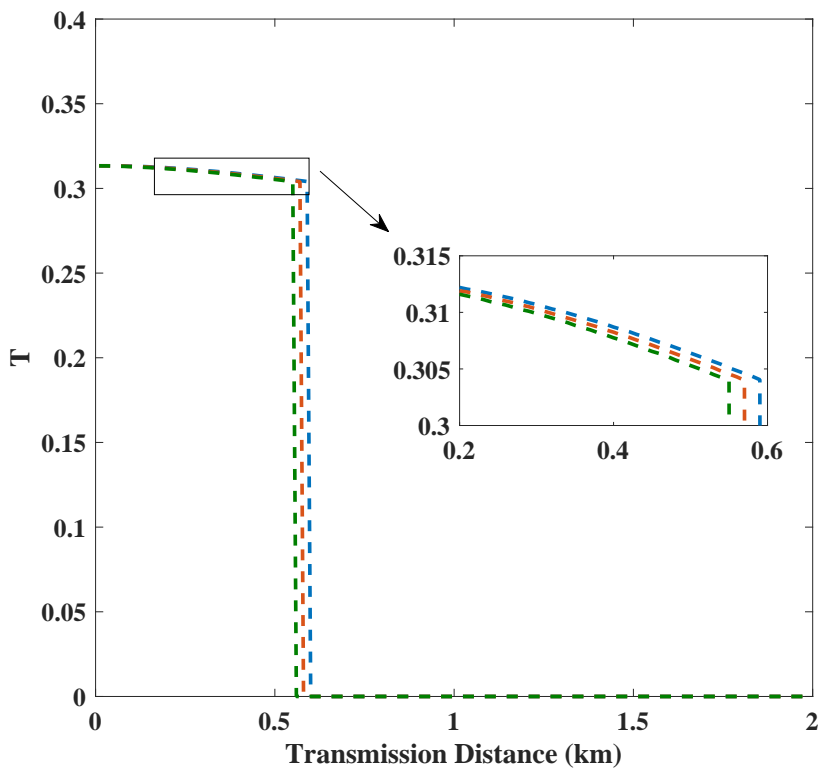

(d)

FIG. 3: (Color online) The secret key rate versus the transmission distance for (a) the extreme asymmetric case and (b) the symmetric case with several different excess noises $\xi_{j}=\xi \in\{0.002,0.0025,0.003\}(j=A, B)$ when optimizing over the transmittance $T$ shown in (c) and (d), respectively. Note that ZPC Protocol and Original Protocol represent the ZPC-involved DM MDI-CVQKD and the original DM MDI-CVQKD. Other parameters are fixed as follows: reconciliation efficiency is $\beta=0.95$, optimal variances $V$ for the original protocol is 1.4 in the extreme asymmetric case (1.5 in the symmetric case), optimal variances $V$ for the ZPC protocol is 2.5 in the extreme asymmetric case (2.6 in the symmetric case).

means that the value of $V$ can be expanded appropriately by using the ZPC operation on DM MDI-CVQKD. To understand this point, for the given $\beta=0.95$, Figs. 2 (a) and 2(b) show the secret key rates as a function of the variance $V$ with the extreme asymmetric and symmetric cases, when optimizing over the transmittance $T$ shown in Figs. 2(c) and 2(d), respectively. As for the original DM MDI-CVQKD protocols (solid line), includ- ing the extreme asymmetric and symmetric cases, the optimal areas of $V$ are gradually compressed with the decrease of the transmission distance, whereas for the ZPCinvolved DM MDI-CVQKD protocols (dashed line), the secret key rate decreases much more slowly with the increase of $V$. In other words, the ZPC-involved protocols have much larger optimal areas of $V$ than the original protocols, which implies that employing the ZPC opera- 


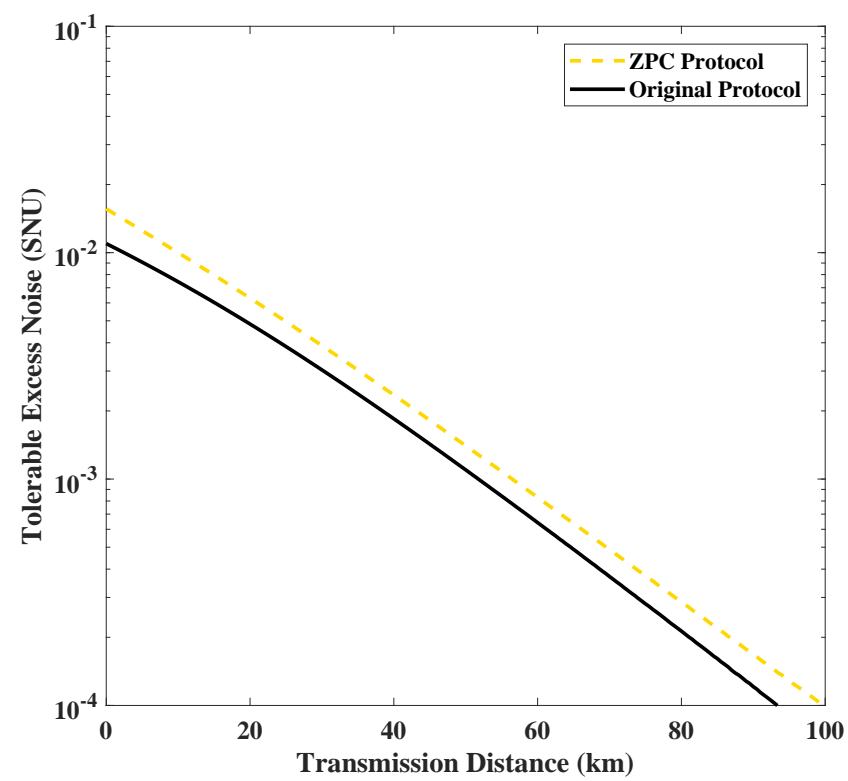

(a)

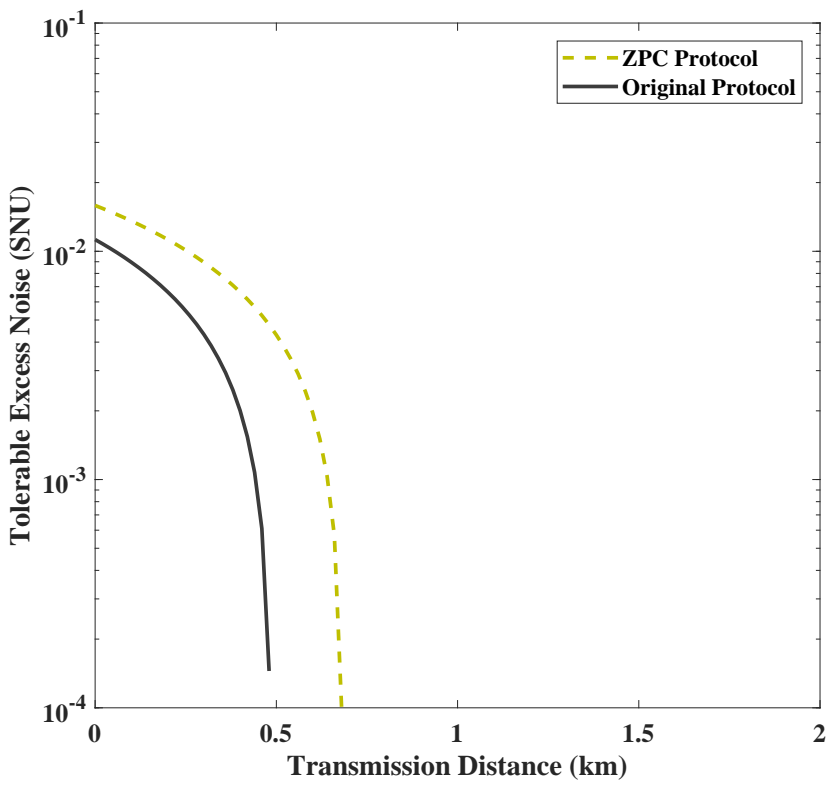

(b)

FIG. 4: (Color online) The tolerate excess noise versus the transmission distance for (a) the extreme asymmetric case and (b) the symmetric case when optimized over the transmittance $T$. Note that ZPC Protocol and Original Protocol represent the ZPC-involved DM MDI-CVQKD and the original DM MDI-CVQKD. Other parameters are fixed as follows: reconciliation efficiency is $\beta=0.95$, optimal variances $V$ for the original protocol is 1.4 in the extreme asymmetric case (1.5 in the symmetric case), optimal variances $V$ for the ZPC protocol is 2.5 in the extreme asymmetric case (2.6 in the symmetric case).

tion on the DM MDI-CVQKD systems would bring about more flexible and stable performances. We also notice that, when $V$ exceeds a certain threshold, the secret key rate of the ZPC-involved DM MDI-CVQKD system can be higher than that of the original one, which reveals that the usage of the ZPC operation can offer a possible way to increase the secret key rate well. To be specific, for the original protocols, the optimal value of $V$ under the extreme asymmetric case (the symmetric case) is about 1.4 (1.5) with several different transmission distances, e.g., $25 \mathrm{~km}, 30 \mathrm{~km}$ and $35 \mathrm{~km}(0.1 \mathrm{~km}, 0.2 \mathrm{~km}$ and $0.3 \mathrm{~km})$, as described in Ref. [46]. While for the proposed protocols, we find that the correspondingly optimal $V$ under the extreme asymmetric case (the symmetric case) is about 2.5 (2.6). As shown in Figs. 2(c) and 2(d), the corresponding transmittances are, respectively, given by $T \in\{0.275,0.266,0.258\}$ and $T \in\{0.312,0.311,0.310\}$ so that the modulated variance $\widetilde{V}_{M}$ of the proposed protocols can also satisfy the constraint $\widetilde{V}_{M}<0.5$. Thus, in the following, we shall show the best performance of the DM MDI-CVQKD system when taking into account these optimal variances.

\section{B. Security analysis}

The security of the traditional QKD systems can be reflected from three aspects, i.e., the secret key rate, the tolerable excess noise and the transmission distance. In what follows, we focus on the security analysis of the ZPC-involved DM MDI-CVQKD system, including the extreme asymmetric and symmetric cases, compared with the original DM MDI-CVQKD.

In Figs. 3(a) and 3(b), we illustrate the secret key rate of both protocols versus the transmission distance with the special two cases, i.e., the extreme asymmetric case and the symmetric case, optimized over the transmittance $T$ depicted in Figs. 3(c) and 3(d), respectively. The solid lines denote that the original protocols, which can be surpassed by the protocols using the ZPC operation in terms of the secret key rate and the transmission distance. Compared with the original protocol, the transmission distance of the proposed protocol is closer to the PLOB bound [55] that represents the ultimate limit of repeaterless communications. One reason is that, by regulating the transmittance $T$, the optimal variance $V$ for the latter can be obtained in the long-distance communication, and can be bigger than the former case $(V=1.4$ under the extreme asymmetric case and $V=1.5$ under the symmetric case). The other is that the ZPC operation can be indeed viewed as a noiseless linear attenuation, which has been used for improving the transmission distance [56]. In addition, when comparing with the extreme asymmetric case, the performance of both protocols in the symmetric case is very poor under the same parameters, because the gap of the excess noise between the extreme asymmetric and symmetric cases becomes large with the increase of the transmission dis- 


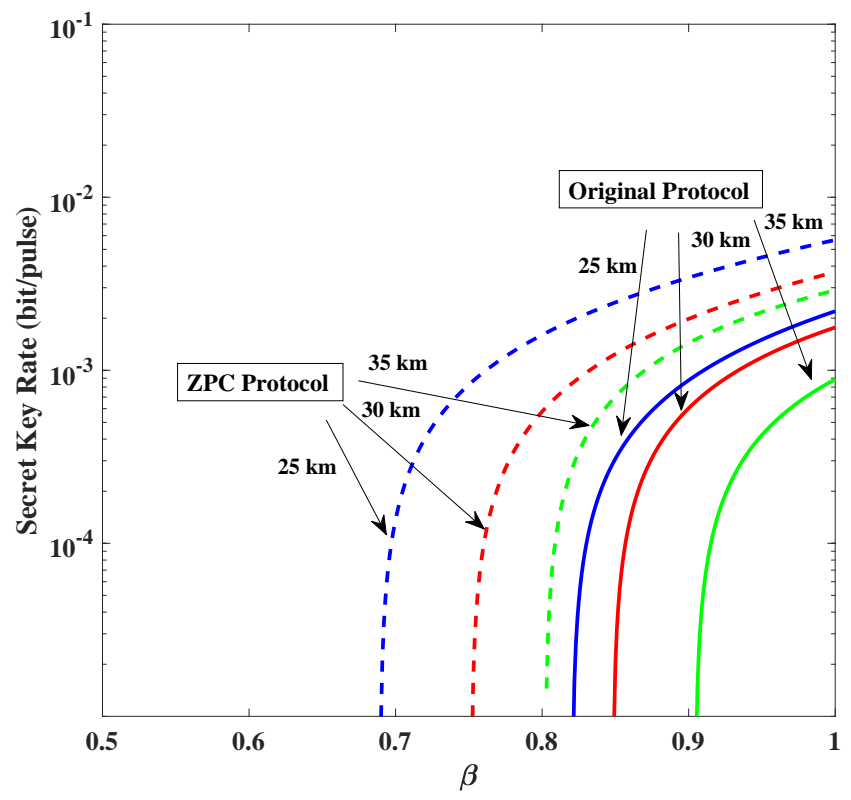

(a)

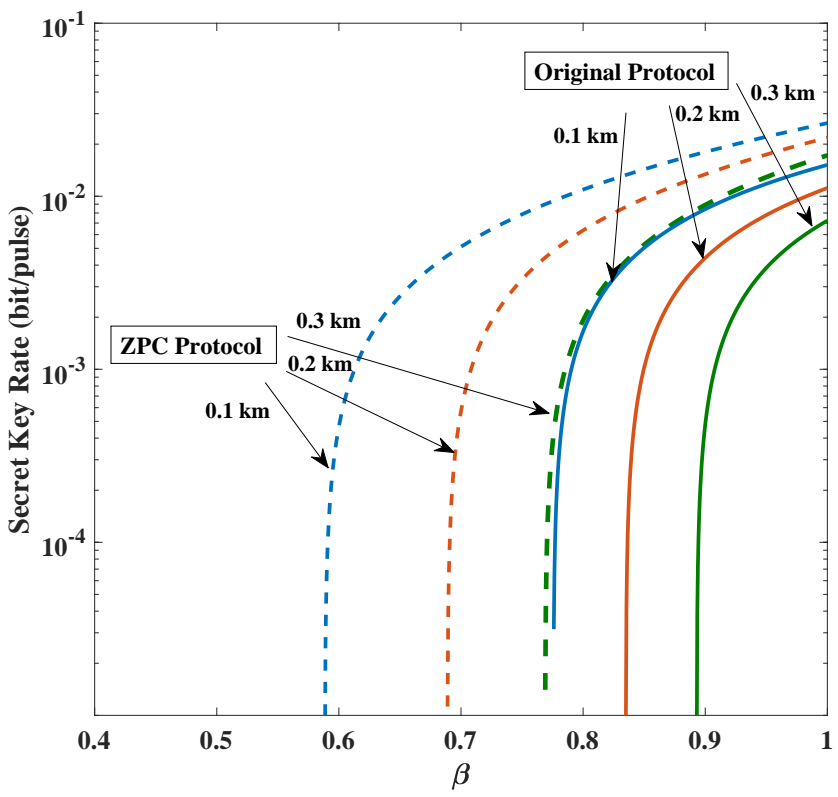

(b)

FIG. 5: (Color online) The secret key rate versus the reconciliation efficiency $\beta$ for (a) the extreme asymmetric case and (b) the symmetric case with with several different transmission distances e.g., $25 \mathrm{~km}, 30 \mathrm{~km}, 35 \mathrm{~km}$ and $0.1 \mathrm{~km} 0.2 \mathrm{~km}, 0.3 \mathrm{~km}$ when optimized over the transmittance $T$, respectively. Note that ZPC Protocol and Original Protocol represent the ZPC-involved DM MDI-CVQKD and the original DM MDI-CVQKD. Other parameters are fixed as follows: excess nosie is $\xi_{j}=\xi=0.002(j=A, B)$, optimal variances $V$ for the original protocol is 1.4 in the extreme asymmetric case (1.5 in the symmetric case), optimal variances $V$ for the $\mathrm{ZPC}$ protocol is 2.5 in the extreme asymmetric case (2.6 in the symmetric case).

tance [46]. We find that the performances of the above DM MDI-CVQKD systems, even with the ZPC operation, are affected by the increase of $\xi=0.002,0.0025$ and 0.003 , especially in terms of the maximal transmission distance. It reveals that the above-mentioned protocols are sensitive to the excess noise. To further understand this point, Figs. 4(a) and 4(b) show the tolerable excess noise as a function of the transmission distance with the extreme asymmetric and symmetric cases, when taking into account each possible transmittance $T$. We find that, with the help of the ZPC operation, the tolerable excess noise of the proposed protocol can be further improved, which implies that under the same acceptable excess noise, the ZPC-involved DM MDI-CVQKD protocol has obvious advantages over the original protocol in terms of the maximal transmission distance. In particular, when $\xi \approx 0.001$, the proposed protocol is able to support a robust DM MDI-CVQKD system over long distance about $56 \mathrm{~km}$. However, there is a serious problem that, as for the extreme asymmetric and symmetric cases, once the excess noise of both protocols is greater than a certain threshold (about 0.015 for the proposed protocol and 0.011 for the original protocol), we can not achieve the positive secret key rate. To solve this problem, fortunately, a new two-way CVQKD to improve robustness against excess noise has been proposed [57]. We also notice that, with the increase of transmission distance, the excess noise of both protocols in the symmetric case falls faster than that in the extreme asymmetric one, thereby making these protocols perform worse than the extreme asymmetric case with respect to long-distance communication.

The reconciliation efficiency $\beta$, on the other hand, is an important indicator of extracting secret key information. As shown in Figs 5(a) and 5(b), we give the secret key rate versus the reconciliation efficiency $\beta$ with the extreme asymmetric and symmetric cases, respectively. We find that, in the extreme asymmetric case, the available range of $\beta$ for both the proposed protocol (dashed lines) and the original protocol (solid lines) narrows as the transmission distance increases. Moreover, for the given transmission distance, the performance of the ZPCinvolved DM MDI-CVQKD system is always better than that of the original one with respect to the secret key rate and the tolerant of $\beta$. This phenomenon may be caused by two aspects that the optimal $V$ can be legitimately expanded by using the ZPC operation on the DM MDI-CVQKD system (shown in Figs. 2(a) and 2(b)) and the system with discrete modulation works well at the extremely low signal-to-noise ratio with a high-efficiency error correction code in the reconciliation process. These results are also true for the symmetric case, as illustrated in Fig. 5(b). In this sense, it reveals that compared with the original protocol, the ZPC-involved DM MDICVQKD protocol in the symmetric case is more beneficial for short-distance communication, especially under the 
low reconciliation efficiency.

\section{CONCLUSION}

We have suggested the performance improvement of the MDI-CVQKD with discrete modulation by performing the ZPC operation. We focus on the conventional four-state scheme as the representation of the DM-based CVQKD system. Due to the benefits of a high-efficiency error correction code in the reconciliation process, this four-state-based scheme may offers a possible way to extend the maximal transmission distance. In the context of an asymptotic-regime security analysis including the extreme asymmetric and symmetric cases, our results under the same accessible parameters show that the secret key rate of the ZPC-involved DM MDI-CVQKD protocol can be increased, compared with the original protocol. In addition, the performance of the ZPC-involved protocol is always superior to that of the original one in terms of the maximal transmission distance. Furthermore, we find that our protocol enables the DM MDICVQKD system to tolerate more lower reconciliation efficiency. However, the tolerable excess noise of the both protocols, especially for the symmetric case, decreases with the increase of the transmission distance, which re- strains the effects of extending the security transmission distance. Fortunately, the two-way CVQKD protocol to tolerate more excess noise than the one-way protocol was first proposed by S. Pirandola [57], which paves the way for our future investigations in the development of lengthening the security transmission distance.

\section{Acknowledgments}

This work was supported by the National Natural Science Foundation of China (Grant Nos. 61572529, 61821407, 11964013, 11664017), the Outstanding Young Talent Program of Jiangxi Province (Grant No. 20171BCB23034), the Training Program for Academic and Technical Leaders of Major Disciplines in Jiangxi Province, the Postgraduate Scientific Research Innovation Project of Hunan Province (Grant No. CX20190126) and the Postgraduate Independent Exploration and Innovation Project of Central South University (Grant No. 2019zzts070).
[1] H. K. Lo, M. Curty and K. Tamaki, Secure quantum key distribution, Nat. Photonics 8, 595-604 (2014).

[2] B. Korzh, C. C. W. Lim, R. Houlmann, N. Gisin, M. J. Li, D. Nolan, B. Sanguinetti, R. Thew and H. Zbinden, Provably secure and practical quantum key distribution over 307 km of optical fibre, Nat. Photonics 9, 163-168 (2015).

[3] N. Gisin, Gregoire Ribordy, Wolfgang Tittel, and Hugo Zbinden, Quantum cryptography, Rev. Mod. Phys. 74, 145 (2002).

[4] S. L. Braunstein and P. van Loock, Quantum information with continuous variables, Rev. Mod. Phys. 77, 513 (2005).

[5] V. Scarani, H. Bechmann-Pasquinucci, N. J. Cerf, M. Dušek, N. Lutkenhaus, and M. Peev, The security of practical quantum key distribution, Rev. Mod. Phys. 81, 1301 (2009).

[6] A. K. Ekert, Quantum cryptography based on Bell's theorem, Phys. Rev. Lett. 67, 661 (1991).

[7] C. Weedbrook, S. Pirandola, R. Garcia-Patron, N. J. Cerf, T. C. Ralph, J. H. Shapiro, and S. Lloyd, Gaussian quantum information, Rev. Mod. Phys. 84, 621 (2012).

[8] P. Jouguet, and S. Kunz-Jacques, and A. Leverrier, and Grangier, P. and E. Diamanti, Experimental demonstration of long-distance continuous-variable quantum key distribution, Nat. Photonics. 7, 378-381 (2013).

[9] F. Grosshans and P. Grangier, Continuous variable quantum cryptography using coherent states, Phys. Rev. Lett. 88, 057902 (2002).

[10] D. Huang, P. Huang, D. K. Lin, C. Wang, and G. H. Zeng, High-speed continuous-variable quantum key distribution without sending a local oscillator, Opt. Lett. 40, 3695-
3698 (2015).

[11] Y. J. Shen, X. Peng, J. Yang, and H. Guo, Continuousvariable quantum key distribution with Gaussian source noise, Phys. Rev. A 83, 052304 (2011).

[12] B. Qi, P. Lougovski, R. Pooser, W. Grice, and M. Bobrek, Generating the Local Oscillator "Locally" in ContinuousVariable Quantum Key Distribution Based on Coherent Detection, Phys. Rev. X 5, 041009 (2015).

[13] R. G. Patron and N. J. Cerf, Unconditional optimality of Gaussian attacks against continuous-variable quantum key distribution, Phys. Rev. Lett. 97, 190503 (2006).

[14] F. Furrer, T. Franz, M. Berta, A. Leverrier, V. B. Scholz, M. Tomamichel, and R. F. Werner, Continuous variable quantum key distribution: Finite-key analysis of composable security against coherent attacks, Phys. Rev. Lett. 109, 100502 (2012).

[15] Y, C. Zhang, Z. Y. Li, Z. Y. Chen, C. Weedbrook, Y. J. Zhao, X. Y. Wang, Y. D. Huang, C. C. Xu, X. X. Zhang, Z. Y. Wang, M. Li, X. Y. Zhang, Z. Y. Zheng, B. J. Chu, X. Y. Gao, N. Meng, W. W. Cai, Z. Wang, G. Wang, S. Yu, and H. Guo, Continuous-variable QKD over $50 \mathrm{~km}$ commercial fiber, Quantum Sci. Technol. 4, 035006 (2019).

[16] X. C. Ma, S. H. Sun, M. S. Jiang, and L. M. Liang, Local oscillator fluctuation opens a loophole for Eve in practical continuous-variable quantum-key-distribution systems, Phys. Rev. A 88, 022339 (2013).

[17] X. C. Ma, S. H. Sun, M. S. Jiang, and L. M. Liang, Wavelength attack on practical continuous-variable quantumkey-distribution system with a heterodyne protocol, Phys. Rev. A 87, 052309 (2013).

[18] J. Z. Huang, C. Weedbrook, Z. Q. Yin, S. Wang, H. W. Li, 
W. Chen, G. C. Guo, and Z. F. Han, Quantum hacking of a continuous-variable quantum-key-distribution system using a wavelength attack, Phys. Rev. A 87, 062329 (2013).

[19] H. K. Lo, M. Curty, and B. Qi, Measurement-deviceindependent quantum key distribution, Phys. Rev. Lett. 108, 130503 (2012).

[20] S. Pirandola, C. Ottaviani, G. Spedalieri C. Weedbrook, S. L. Braunstein, S. Lloyd, T. Gehring, C. S. Jacobsen, and U. L. Andersen, High-rate measurement-device-independent quantum cryptography, Nat. Photon. 9, 397 (2015).

[21] X. Y. Zhang, Y. C. Zhang, Y. J. Zhao, X. Y. Wang, S. Yu, and H. Guo, Finite-size analysis of continuous-variable measurement-device-independent quantum key distribution, Phys. Rev. A 96, 042334 (2017).

[22] Z. Y. Li, Y. C. Zhang, F. H. Xu, X. Peng, and H. Guo, Continuous-variable measurement-device-independent quantum key distribution, Phys. Rev. A 89, 052301 (2014).

[23] X. C. Ma, S. H. Sun, M. S. Jiang, M. Gui, and L. M. Liang, Gaussian-modulated coherent-state measurement-deviceindependent quantum key distribution, Phys. Rev. A 89, 042335 (2014).

[24] C. Lupo, C. Ottaviani, P. Papanastasiou, and S. Pirandola, Continuous-variable measurement-device-independent quantum key distribution: Composable security against coherent attacks, Phys. Rev. A 97, 052327 (2018).

[25] Y. C. Zhang, Z. Y. Li, S. Yu, W. Y. Gu, X. Peng, and H. Guo, Continuous-variable measurement-deviceindependent quantum key distribution using squeezed states, Phys. Rev. A 90, 052325 (2014).

[26] X. Y. Zhang, Y. C. Zhang, Y. J. Zhao, X. Y. Wang, S. Yu, and $\mathrm{H}$. Guo, Finite-size analysis of continuous-variable measurement-device-independent quantum key distribution, Phys. Rev. A 96, 042334 (2017).

[27] P. Papanastasiou, C. Ottaviani, and S. Pirandola, Finitesize analysis of measurement-device-independent quantum cryptography with continuous variables, Phys. Rev. A 96, 042332 (2017).

[28] Z. Y. Chen, Y. C. Zhang, G. Wang, Z. Y. Li, and H. Guo, Composable security analysis of continuous-variable measurement-device-independent quantum key distribution with squeezed states for coherent attacks, Phys. Rev. A 98, 012314 (2018).

[29] Y. D. Wu, J. Zhou, X. B. Gong, Y. Guo, Z. M. Zhang, and G. Q. He, Continuous-variable measurement-deviceindependent multipartite quantum communication, Phys. Rev. A 93, 022325 (2016).

[30] H. X. Ma, P. Huang, D. Y. Bai, S. Y. Wang, W. S. Bao, and G. H. Zeng, Continuous-variable measurement-deviceindependent quantum key distribution with photon subtraction, Phys. Rev. A 97, 042329 (2018).

[31] Y. J. Zhao, Y. C. Zhang, B. J. Xu, S. Yu, and H. Guo, Continuous-variable measurement-device-independent quantum key distribution with virtual photon subtraction, Phys. Rev. A 97, 042328 (2018).

[32] P. Wang, X. Y. Wang, and Y. M. Li, Continuous-variable measurement-device-independent quantum key distribution using modulated squeezed states and optical amplifiers, Phys. Rev. A 99, 042309 (2019).

[33] Y. Guo, W. Ye, H. Zhong, and Q. Liao, Continuous-variable quantum key distribution with non-Gaussian quantum catalysis, Phys. Rev. A 99, 032327 (2019).

[34] W. Ye, H. Zhong, Q. Liao, D. Huang, L. Y. Hu, and Y. Guo, Improvement of self-referenced continuous-variable quantum key distribution with quantum photon catalysis, Opt. Express 27, 17186-17198 (2019).

[35] L. Y. Hu, J. N. Wu, Z. Y. Liao, and M. S. Zubairy, Multiphoton catalysis with coherent state input: Nonclassicality and decoherence, J. Phys. B: At. Mol. Phys. 49, 175504 (2016).

[36] A. I. Lvovsky and J. Mlynek, Quantum-optical catalysis: generating nonclassical states of light by means of linear optics, Phys. Rev. Lett. 88, 250401 (2002).

[37] W. D. Zhou, W. Ye, C. J. Liu, L. Y. Hu, and S. Q. Liu, Entanglement improvement of entangled coherent state via multiphoton catalysis, Laser Phys. Lett. 15, 065203 (2018).

[38] L. Y. Hu, Z. Y. Liao, and M. S. Zubairy, Continuousvariable entanglement via multiphoton catalysis, Phys. Rev. A 95, 012310 (2017).

[39] W. Ye, H. Zhong, X. D. Wu, L. Y. Hu, and Y. Guo, Continuous-variable measurement-device-independent quantum key distribution via quantum catalysis, arXiv:1907.03383v2 (2019).

[40] T. J. Richardson, M. A. Shokrollahi, and R. Urbanke, Design of capacity-approaching irregular low-density paritycheck codes, IEEE Trans. Inf. Theory 47, 619 (2001).

[41] A. Leverrier, R. Alleaume, J. Boutros, G. Zemor, and P. Grangier, Multidimensional reconciliation for a continuous-variable quantum key distribution, Phys. Rev. A 77, 042325 (2008).

[42] A. Leverrier and P. Grangier, Unconditional security proof of long-distance continuous-variable quantum key distribution with discrete modulation, Phys. Rev. Lett. 102, 180504 (2009).

[43] A. Leverrier and P. Grangier, Continuous-variable quantum-key-distribution protocols with a non-Gaussian modulation, Phys. Rev. A 83, 042312 (2011).

[44] P. Huang, J. Fang, and G. H. Zeng, State-discrimination attack on discretely modulated continuous-variable quantum key distribution, Phys. Rev. A 89, 042330 (2014).

[45] Y. Shen, H. X. Zou, L. Tian, P. X. Chen, and J. M. Yuan, Experimental study on discretely modulated continuousvariable quantum key distribution, Phys. Rev. A 82, 022317 (2010).

[46] H. X. Ma, P. Huang, D. Y. Bai, T. Wang, S. Y. Wang, W. S. Bao, and G. H. Zeng, Long-distance continuous-variable measurement-device-independent quantum key distribution with discrete modulation, Phys. Rev. A 99, 022322 (2019).

[47] S. Ghorai, P. Grangier, E. Diamanti, and A. Leverrier, Asymptotic security of continuous-variable quantum key distribution with a discrete modulation, Phys. Rev. X 9, 021059 (2019).

[48] W. Ye, Y. Guo, Y. Xia, H. Zhong, H. Zhang, J. Z. Ding, and L. Y. Hu, Discrete modulation continuous-variable quantum key distribution based on quantum catalysis, Acta Phys. Sin. 69, 060301 (2020).

[49] J. Yang, B. J. Xu, X. Peng, and H. Guo, Four-state continuous-variable quantum key distribution with long secure distance, Phys. Rev. A 85, 052302 (2012).

[50] M. Navascues, F. Grosshans, and A. Acin, Optimality of Gaussian attacks in continuous-variable quantum cryptography, Phys. Rev. Lett. 97, 190502 (2006).

[51] S. Pirandola, Entanglement reactivation in separable environments, New J. Phys. 15, 113046 (2013).

[52] H. Zhang and G. Q. He, Improving the performance of the four-state continuous-variable quantum key distribu- 
tion by using optical amplifiers, Phys. Rev. A 86, 022338 (2012).

[53] Y. C. Zhang, Z. Y. Li, C. Weedbrook, S. Yu, W. Y. Gu, M. Z. Sun, X. Peng, and H. Guo, Improvement of two-way continuous-variable quantum key distribution using optical amplifiers, J. Phys. B: At. Mol. Opt. Phys. 47, 035501 (2014).

[54] M. M. Wolf, G. Giedke, and J. I. Cirac, Extremality of Gaussian quantum states, Phys. Rev. Lett. 96, 080502 (2006).

[55] S. Pirandola, R. Laurenza, C. Ottaviani, and L. Banchi,
Fundamental limits of repeaterless quantum communications, Nat. Commun. 8, 15043 (2017).

[56] J. Fiurasek and N. J. Cerf, Gaussian postselection and virtual noiseless amplification in continuous-variable quantum key distribution. Phys. Rev. A 86(6), 060302(R) (2012).

[57] S. Pirandola, S. Mancini, S. Lloyd, and S. L. Braunstein, Continuous-variable quantum cryptography using two-way quantum communication, Nat. Phys. 4, 726-730 (2008). 